\title{
Thinking on the Planning and Design of Environmental Landscape in Qiang Region
}

\author{
Jundong Ye \\ School of Art, Aba Teachers College, Wenchuan, China
}

\begin{abstract}
Based on the analysis of the design of environmental landscape architecture in Qiangzhai Style, it is proposed that the design of landscape architecture should be based on computer-aided design technology. Landscape architecture design integrates many disciplines, and any good building usually includes two aspects of internal and external space design. The design idea is the premise of deciding whether the Qiang landscape architecture design can be done well or not. It needs more understanding of the Qiang architecture, culture and art to do the related design well. Cultural creativity is also an important part of the strategy of Rural Revitalization and the construction of characteristic villages in Qiang nationality areas
\end{abstract}

\section{Introduction}

Qiang is a minority nationality with a long history in China. Now it mainly lives in Aba, Sichuan Province. At present, rural areas in Qiang nationality area are generally developing economy vigorously, and various rural tourism and farmhouse pleasure are rising. Researchers should pay attention to the strategy of rural revitalization and the construction of characteristic villages in Qiang nationality area. A resident of a Qiang village in Western Sichuan has developed a landscape architecture project which integrates catering, leisure and sightseeing. It mainly constructs a landscape architecture with Chinese style and Qiang architectural style. The first floor is catering, and the second and third floors are accommodation. In front of the house, a narrow and winding garden yard was built, in which several pavilions with some Chinese garden style and artificial landscape pools were built, and a lot of greening was done. On the roof of the main landscape building and at the gate of the courtyard, the villager put up a sign called "Qiang Village Style" and held a number of Qiang style dances. The guests ate a lively scene of roasting whole sheep, which caused many people to watch. However, over the years, not many visitors have come to the "Qiang Village Style " for recreation or sightseeing. On an occasional occasion, the author learns that the villager first found a local well-known artist to realize his idea, design drawings, that is, several hand-painted effect drawings, and then the villagers found people engaged in construction to complete the construction. The first impression of this landscape architecture is not very much like Qiang architecture, especially the lack of Qiang culture elements in interior decoration. The whole indoor and outdoor environment design makes people feel less beautiful, not attractive, and the use function of the building is not considered. To do a good job in the planning and design of environmental landscape in Qiang nationality area, designers should think from many aspects.

\section{Make full use of computer aided design technology}

With the rapid development of computer science and technology, computer-aided technology for environmental landscape planning and design is becoming more and more advanced. Geographic Information System (GIS) and Virtual Reality (VR) have become important technologies in landscape planning and design. CAD, 3DMAX, SKETCHUP, VARY, ENSCAPE, LUMION, PHOTOSHOP, VR virtual reality technology and GIS geographic information system have become the technologies widely used in landscape design. The earliest computer aided design technology and platform used in graphic drawing technology is CAD, which has been widely used in urban planning, architectural design and industrial design, and also in landscape design, bringing new ideas and concepts to designers. The advantage of CAD is that the drawing is accurate. Now, it is mainly used for drawing detailed construction drawings, and also for three-dimensional modeling and 
performance. Compared with traditional hand-drawn design, it can better use the design intentions in actual construction, avoid the similarities of landscape design schemes, and faster than traditional manual drawing. In addition, the modeling and rendering software, as well as other software continue to bring forth new field of vision and progress. Landscape planning and design has achieved the convergence of science and aesthetics, and has become a comprehensive and systematic design discipline. [1] Therefore, in order to do a good job of landscape planning and design in Qiang nationality area, designers should make full use of computer-aided design technology, in order to have a brilliant design plan and satisfactory performance.

\section{Landscape architecture and design in Qiang Nationality Area}

\subsection{Traditional landscape architecture of Qiang Nationality}

The Qiang people generally live in their stockades. Their traditional buildings are stone and stone towers, and some of them are built entirely from loess clay. There are three or four stories in a blockhouse. The traditional stone blockhouse of the Qiang nationality has an inclination angle of about 10 degrees. The walls inside the house are vertical, and the walls outside the house are gradually contracting from bottom to top, forming a narrow trapezoid on the width below and above. The whole building is similar to the overlay bucket. The main building materials are stone, clay and wood. The wall structure receives a lot of points in the construction technology. Qiang architecture also includes temples, bridges, trestles, official villages, workshops and so on. From the beginning to the end of traditional Qiang architecture construction, there are no drawings and brackets, and no suspension layouts, all based on superb skills and experience. [2] In addition, the buildings are not made of reinforced concrete, but local materials. Some buildings did not collapse in the earthquake, and still stand erect to maintain the original landscape. Qiang people's Shicheng dwelling houses, the blockhouse is a continuation of the style of dry railings. [3] Traditional Qiang architecture shows a simple and natural image, forming a unique and spectacular landscape.

\subsection{Comprehensive environmental discipline}

Landscape architecture is a combination of natural and artificial elements through art and science. Apart from house buildings, flower racks, doors, wooden floors, grape racks, pavilions, corridors, platforms, rockeries, pools, fences, waterscapes, fountains, lawns, swing roads are the contents of landscape architecture. Landscape architecture design is similar to landscape design and environmental design, but with different names, its content is very extensive. Landscape architecture design is also landscape design. In addition to the so-called gardening, greening and gardening, it also includes the planning, management and utilization of ecological land and the development of territorial landscape, which is a comprehensive environmental discipline. Landscape Architecture Design is a multi-disciplinary design field which integrates architecture, gardening, planning and other disciplines. Landscape architecture usually appears in scenic spots, parks, squares and other landscape sites, or it has the function of landscape marking itself. It can be said that it is a landscape, but also has the role of landscape. Landscape architecture differs from ordinary architecture in that it combines environment with culture and pays attention to them.

\section{Qiang's interior furnishings and interior and exterior environment design}

\subsection{Qiang Interior Furnishings}

Qiang watchtower indoor bottom keep the cattle and lay asde farming tools; the middle floor has bedrooms, storage rooms, fire ponds, Guozhuang and shrines, respectively, for people to live in the floor; the upper floor for the Qiang "Baishi God" and grain storage; the roof of the house is a platform, mainly used for sunning food, while entertainment and leisure use. The Qiang people's interior furnishings have been influenced by the Han people. The interior reflects some characteristics of the Han people's interior. However, there are fire ponds in every family's house, and shrines such as "the God of corner" and "the master of heaven and earth". Walking into the interior of the landscape architecture of "Qiang village amorous feelings", we can see the windows, doors and indoor room doors which are the neoclassical traditional style in the Han nationality area of China. There is no cultural connotation related to Qiang nationality in the interior. It's just that the appearance of the landscape architecture has a little something to do with the Qiang style, but it's a little simple. Although the interior furnishing design of "Qiang village amorous feelings" landscape architecture does not necessarily follow the traditional Qiang interior design, but it can not be in the interior furnishing or decoration without any Qiang style characteristics.

\subsection{Interior environment design}

Indoor and outdoor environment design is an integral part of landscape architecture design. Usually, a good building contains two basic contents interior and outdoor space design. An indoor space environment will have different effects after completion, which is caused by the different styles of interior architectural design schemes. Most experts believe that the interior design is actually the interior space environment design, is the deepening of architectural design, is the interior work, study, life and other necessary environmental space for the composition of the desired design. Generally speaking, interior decoration are only the work and means to realize the partial design of interior environment design. 
Interior environment design involves furniture furnishing, texture and performance of decoration materials, arts and crafts, greening, gardening and other art fields, and it is a new subject with strong comprehensiveness. It is closely related to architecture, structural engineering, ergonomics, architectural physiology, sociology, building materials, psychology, folklore and other disciplines. Therefore, the interior environment design not only considers the problems of the ceiling, the ground and the wall of the building space, but also uses interdisciplinary knowledge to construct the space environment comprehensively in all directions and at many levels. Such as the use of knowledge of plane composition, three-dimensional composition and spatial composition, as well as the principles and material means of light and shadow, reflection and color change, illusion, etc. to change the size and scale of space, different psychological feelings, create the required style and environmental atmosphere.

\subsection{Outdoor environment design}

The external environment of the building is a reflection of the technology and art of a nation and an era, and also a true portrayal of the residents' life style, ideology and values. This kind of reflection of cultural regionality, epochality and comprehensiveness is incomparable to any other environment or individual thing. This is because the external environment of the building contains more human impressions reflecting culture, and new content is added every moment. The external environment of group buildings is often a symbol of a city, a region, or even a nation or a national culture. [4] For example, Walian Residence, designed by Malaysian Chinese architects in 1982, has a 15-metre-high atrium with three-storey roof and projecting air windows, which can be ventilated from front to back. Deep eaves make people live in a pleasant environment with good ventilation and no sunshine. Part of the atrium is built above the pool. There is a swimming pool above the pool, just above the surface of the water. The inclined wall beside it makes the water in the pool pour into the lower pool like a waterfall. The wood structure of Walian residential houses is very delicate, which represents the modern buildings with local interest that had begun to be popular at that time. This style is especially popular in tourist hotels. [5] In fact, this building is a landscape building.

From this, we can see that indoor and outdoor environmental design is an integral part of landscape architecture design, which is an important component of landscape architecture design. If the landscape architecture of villages in Qiang area develops economy, without excellent interior and exterior environment design, the landscape architecture will be eclipsed, will not be noticeable and loved by people.

\section{How to design landscape architecture of Qiang Nationality}

\subsection{Design ideas decide design}

How to do a good job in Qiang Landscape Architecture Design is actually decided by the design idea. The ideas mentioned here are mainly the professional knowledge acquired and the comprehensive thinking ability formed by the relevant knowledge of traditional Qiang culture. Without abundant professional knowledge, no matter how good the idea is, it will be difficult to achieve good wishes, so people will not like the final landscape architecture and its environment. Environmental planning, implication, concept, theme, form, space, streamline, aesthetic, artistic conception, material, color and so on should be considered in the design thought. Landscape architecture can also be said to be architectural landscape, which belongs to the category of landscape planning and design. Modern landscape planning and design is a subject specialty which faces to life, is close to life and is very popular. But this does not mean that the threshold of discipline specialty is popular and secular. On the contrary, for the personnel engaged in this subject specialty, to fulfill their mission of discipline specialty, the need is comprehensive. Scientific knowledge, profound life experience and touching artistic imagination. [6] Architectural Design of Individual or Group Design

\section{References}

1. Liu Haiyuan. Application of Computer Aided Technology in Landscape Architecture Planning and Design: Taking Xinghua Park in Gaocheng District as an example [D]. Hebei University of Economics and Trade, 2018:8.

2. Jiang Bin, Zhang Yuan. Research on the Protection and Development of Traditional Culture of Qiang [J]. Journal of Southwest Minzu University (Humanities and Social Sciences Edition), 2009(4):20.

3. Zhang Ben. Study on the Artistic Arts of Qiang ethnic group [M]. Beijing: Tsinghua University Press, 2013:137.

4. Qian Jian, Song Lei. Design of the external environment of the building [M]. Shanghai: Tongji University Press, 2001:12-13.

5. Luo Xiaomo. History of Modern Foreign Architecture [M]. Beijing: China Construction Industry Press, 2004:294-295.

6. Xu Qing. Landscape Design Science [M]. Shanghai: Tongji University Press, 2014: 65, 67.

7. Xu Qing. Landscape Design Science [M]. Shanghai: Tongji University Press, 2014: 65, 67.

8. Zhang Huihui. Research on Public Art Design in Tourist Attractions of Qiang --- Using Buwa Village as An Example [D]. Southwest Jiaotong University, 2012. 